\title{
Progression-free or overall survival...revisited in BOLERO-2
}

I have had the privilege to comment on the relative merits of progression-free survival (PFS) and overall survival (OS) as endpoints, in an editorial in this journal, more than 2 years ago. ${ }^{[1]}$ That was on occasion of the then raging debate about withdrawal of approval for breast cancer indication from bevacizumab by United States Food and Drug Administration (US FDA). More recently, everolimus has begun to be used in patients with metastatic breast cancer (MBC) in combination with exemestane, based on the results of BOLERO-2 trial. ${ }^{[2]}$ In this study, patients with estrogen receptor positive, human epidermal growth factor receptor 2 negative MBC who were refractory to letrozole or anastrozole were randomly assigned to everolimus plus exemestane or placebo plus exemestane in a 2:1 ratio. The published results showed that, compared with placebo, everolimus showed a highly significant improvement in radiologically defined PFS using both investigator (6.9 vs. 2.8 months, hazard ratio $[\mathrm{HR}]=0.43$; $95 \%$ confidence interval $[\mathrm{C}], 0.35-0.54 ; P<0.001)$ and independent reviewers' assessment (10.6 vs. 4.1 months, $\mathrm{HR}=0.36 ; 95 \% \mathrm{CI}, 0.27-0.47 ; P<0.001) .{ }^{[2]}$ Based on these data, the US FDA approved everolimus for the treatment of appropriately selected postmenopausal women with advanced breast cancer. There was substantial toxicity in the everolimus arm, including stomatitis, infections, hyperglycemia, fatigue, dyspnea, pneumonitis, and diarrhea. The most common grades 3-4 laboratory abnormalities were lymphopenia, hyperglycemia, anemia, hypokalemia, elevated aspartate transaminase/alanine transaminase, and thrombocytopenia. Fatal adverse reactions occurred in $2 \%$ of patients receiving everolimus compared with $0.4 \%$ of patients receiving placebo. Adverse reactions resulting in permanent treatment discontinuation occurred in $24 \%$ of patients receiving everolimus and $5 \%$ of patients receiving placebo. Dose interruptions or reductions were necessary in $63 \%$ of patients receiving everolimus compared with $14 \%$ of patients receiving placebo. Subsequent reports from the same study showed that everolimus resulted in a significantly prolonged time to deterioration in global

\begin{tabular}{|l|l|}
\hline \multicolumn{2}{|c|}{ Access this article online } \\
\hline Quick Response Code: & Website: \\
\hline & www.jmpo.org \\
\hline & \\
\hline
\end{tabular}

quality of life (QOL) compared with placebo. ${ }^{[3]}$ This was despite the higher rate of treatment discontinuation resulting from adverse effects. Moreover, QOL was preserved at various time points despite toxicity in the everolimus arm.

The OS data from this trial was recently presented at a median follow-up of 39 months when there were sufficient events for an adequately powered analysis based on protocol assumptions. ${ }^{[4]}$ The results failed to demonstrate a significant improvement in OS for everolimus compared with placebo (30.98 months vs. 26.55 months, $H R=0.89$, $95 \% \mathrm{CI}, 0.73-1.10, P=0.14)$. Of special note, crossover to everolimus was not allowed to participants in the control arm thus eliminating one of the commonly advanced arguments for lack of demonstrable OS benefit. Data presented by Dr. Piccart showed that after progression on study treatment, a somewhat higher fraction of subjects in the control arm received chemotherapy and a slightly higher fraction in the everolimus arm received further endocrine therapy.

So how should we consider the results of BOLERO-2 trial? There was an impressive increase in PFS, no significant deterioration in health related QOL (HRQOL) and lack of significant improvement in OS with everolimus. Several questions and considerations arise when we consider the design and implementation of this trial. An objective, dispassionate attempt to answer these questions has the potential to inform many similar conundrums in contemporary oncology research and practice. First, was the control arm of exemestane an adequate comparator in the setting of patients refractory to previous aromatase inhibitors? Many oncologists would prefer to use chemotherapy in this patient population, especially in those with visceral metastases. Would the results be different had a stronger comparator, perhaps one of the chemotherapy options, been used? Second, although OS was not the primary endpoint, it has been stated to be the key secondary objective in published protocol and a corresponding power calculation performed. The trialists hypothesized that treatment with everolimus plus exemestane will result in a clinically meaningful $26 \%$ reduction in the hazard rate for progression (HR $=0.74$, corresponding to a $35 \%$ increase in the median PFS from 3.7 to 5 months), and they powered the study accordingly. Surprisingly, they also hypothesized that adding everolimus to exemestane will result in a 
$26 \%$ reduction in the hazard rate for death $(\mathrm{HR}=0.74$, corresponding to an increase in median survival from 24 to 32.43 months)! The benefit in PFS was expected to translate almost completely into an OS benefit! As it turned out, the benefit in PFS far outstripped the OS effect. Would a larger study have proved an OS benefit? Unfortunately, because of the aforementioned design considerations, this question is likely to remain unanswered. Third, the argument, that a long postprogression survival makes it hard to prove an OS benefit, merits careful consideration. This statement, while being statistically correct, essentially means that small or moderate leads at the end of the first $200 \mathrm{~m}$ of a $2 \mathrm{~km}$ long race do not necessarily guarantee victory to the early leader. In fact, as the history of metastatic trials show, more often than not both the sprinters end up together! Should we then care for an early lead (PFS) when not even one tenth the race is complete? In this context, regulatory agencies and clinicians need to carefully consider whether artificial constructs such as radiologically defined PFS (using closely performed scans) are real indicators of patient benefit. These questions are particularly pertinent because the treatments in question are almost uniformly expensive or toxic or both. Fourth, how should we assimilate the HRQOL data from BOLERO-2? Is the prolongation of time to deterioration in global QOL clinically meaningful? Do patients really prefer a "preserved QOL" in the presence of substantial toxicity when they gain time on CT scans but not in lifespan? Fifth, in the light of all the preceding, how should we evaluate the relative utility of competing therapeutic options like high dose fulvestrant or chemotherapy? Unfortunately, correlative biomarker analyses from the BOLERO-2 study have failed to provide very useful leads. ${ }^{[5]}$ Sixth, regulatory agencies would need to evolve similar standards when evaluating the worth of different treatments such as bevacizumab and everolimus in MBC. Finally and most importantly, should clinicians not carefully consider the balance of evidence before embracing and advocating new therapies and technologies?
Simple as it may sound, this seems extraordinarily difficult to me in an instantly interconnected world, where medium often supplants the message.

On a personal note, this is my last piece as the Editor-inChief of this journal. I have immensely enjoyed my tenure and am sure that the journal will attain new heights under its new editorial stewardship.

Sudeep Gupta

Editor-in-Chief

Tata Memorial Centre, Mumbai, Maharashtra, India E-mail: sudeepgupta04@yahoo.com

\section{REFERENCES}

1. Gupta S. Progression-free or overall... That is the question.... Indian J Med Paediatr Oncol 2010;31:103-4.

2. Baselga J, Campone M, Piccart M, Burris HA $3^{\text {rd }}$, Rugo HS, Sahmoud $\mathrm{T}$, et al. Everolimus in postmenopausal hormonereceptor-positive advanced breast cancer. N Engl J Med 2012;366:520-9.

3. Burris HA $3^{\text {rd }}$, Lebrun F, Rugo HS, Beck JT, Piccart M, Neven P, et al. Health-related quality of life of patients with advanced breast cancer treated with everolimus plus exemestane versus placebo plus exemestane in the phase 3 , randomized, controlled, BOLERO-2 trial. Cancer 2013;119:1908-15.

4. Piccart M, Hortobagyi GN, Campone M, Pritchard KI, Noguchi S, Rugo $\mathrm{H}$, et al. Everolimus Plus Exemestane for Hormone Receptor-Positive $(\mathrm{HR}+)$, Human Epidermal Growth Factor Receptor-2-Negative (HER2-) Advanced Breast Cancer (BC): Overall Survival Results From BOLERO-2. Presented at EBCC-9; 19-21 March 2014; Glasgow, Scotland. Abstract 1 LBA.

5. Hortobagyi GN, Piccart-Gebhart MJ, Rugo HS, Burris HA, Campone $\mathrm{M}$, Noguchi $\mathrm{S}$, et al. Correlation of molecular alterations with efficacy of everolimus in hormone receptorpositive, HER2-negative advanced breast cancer: Results from BOLERO-2. 2013 ASCO Annual Meeting. Abstract LBA509.

How to cite this article: Gupta S. Progression-free or overall survival...revisited in BOLERO-2. Indian J Med Paediatr Oncol 2014;35:1-2.

Source of Support: Nil. Conflict of Interest: None declared. 